\title{
Postural stability in patients with non-specific chronic neck pain: A comparative study with healthy people
}

\author{
Maryam Saadat ${ }^{1}$, Reza Salehi*1, ${ }^{2}$, Hossein Negahban ${ }^{3,4}$, Mohammad Jafar Shaterzadeh ${ }^{1}$, \\ Mohammad Mehravar ${ }^{1}$, Masumeh Hessam ${ }^{1}$
}

\begin{abstract}
Background: Impairment of cervical sensory input in patients with neck pain may disturb postural stability. The purpose of present study was to assess the dynamic postural stability of subjects with chronic neck pain compared to a matched control group.

Methods: In this case-control study, 22 chronic non-specific neck pain and 22 healthy individuals participated. Postural stability was measured with Techno-body Prokin tilting platform. Subjects performed balance tests under two conditions: eyes open and closed. The parameters for assessment of postural stability were total stability index (TSI), anteroposterior stability index (APSI), mediolateral stability index (MLSI), and trunk deviation which demonstrated total trunk sway in medio-lateral and antero-posterior. We used a separate 2 (group) by 2 (postural difficulty) mixed-design analysis of variance (ANOVA) for analysis of postural performance.

Results: There were significant differences between the chronic neck pain and matched control groups in APSI, MLSI, and TSI, $\mathrm{p}<0.001$ in both eyes opened and closed conditions. The trunk deviation was greater for non-specific neck pain in comparison to healthy subjects, $\mathrm{p}<0.05$ in both conditions of eyes open and closed.

Conclusion: The results of this study showed that patients with chronic neck pain have poorer postural control than healthy subjects. The findings suggest that clinicians take into account the importance of dynamic postural stability assessment in patients with chronic non-specific neck pain and consider the application of intervention programs for improvement of the dynamic balance.
\end{abstract}

Keywords: Chronic neck pain, Dynamic stability, Posture, Balance

Copyright $\odot$ Iran University of Medical Sciences

Cite this article as: Saadat M, Salehi R, Negahban H, Shaterzadeh MJ, Mehravar M, Hessam M. Postural stability in patients with non-specific chronic pain: A comparative study with healthy people. Med J Islam Repub Iran. 2018(23 Apr);32:33. https://doi.org/10.14196/mjiri.32.33

\section{Introduction}

Neck pain is an important public health problem, which causes economic cost, absence from work and reduced quality of life (1). Every year, 30 to $50 \%$ of adults, experience neck pain with a higher incidence among women (2). Neck pain is associated with pain, decreased range of motion, changes in muscle function, joint position sense deficit and postural stability disturbance $(3,4)$.

The postural stability is essential for the execution of daily activities and can be assessed in both static and dynamic conditions. Static tests assess individual's ability to

\section{Corresponding author: Dr Reza Salehi, salehi200@yahoo.com}

1. Musculoskeletal Rehabilitation Research Center, Ahvaz Jundishapur University of Medical Sciences, Ahvaz, Iran.

2. Rehabilitation Research Center, and Department of Rehabilitation Management, School of Rehabilitation Sciences, Iran University of Medical Sciences, Tehran, Iran.

3. Department of Physical Therapy, School of Paramedical Sciences, Mashhad University of Medical Sciences, Mashhad, Iran.

4. Orthopedic Research Center, Mashhad University of Medical Sciences, Mashhad, Iran. maintain the body's center of mass within a fixed base of support and dynamic tests assess the ability of individuals to keep the body's center of mass over a moving base of support (5). Both static and dynamic postural stability require integration of inputs from visual, vestibular and proprioceptive systems in order to achieve a motor response (6).

The cervical muscles, especially the suboccipital muscles have a high density of muscle spindles which send rich proprioceptive inputs to the central nervous system which in

$\uparrow$ What is "already known" in this topic:

Altered cervical proprioceptive information in patients with neck pain influences the integration of inputs within the postural control system. Some studies examined postural stability in patients with non-specific neck pain but there is no general agreement in the literature about the decreased postural stability of these patients.

\section{$\rightarrow$ What this article adds:}

Postural control decreased in patients with chronic non-specific neck pain in comparison with healthy subjects. Dynamic stability tests are a good choice to discriminate balance deficits in patients with non-specific chronic neck pain which may be masked under static conditions. 
turn tune spatial orientation and movement of the head in relation to the rest of the body. There are central and reflexive connections between cervical receptors and visual and vestibular systems $(7,8)$. In patients with neck pain, signs and symptoms such as pain, inhibition of deep neck muscles, fatigue, and psychosocial distress can alter cervical mechanoreceptors sensitivity (7). Therefore, it is assumed that a sensory mismatch between abnormal cervical information and vestibular and visual systems in neck pain may disturb postural stability.

Several studies have examined postural stability in patients with non-specific neck pain (9-11). Some of these studies demonstrated a significant difference in postural sway measures in patients with chronic non-specific neck pain in comparison with healthy individuals $(11,12)$. Others did not observe a significant difference $(9,10)$. One possible reason such disagreement in the reported postural sway could be due to different study designs and setups, for instance, decreased postural stability has been shown in elderly patients with neck pain, whereas the effect of aging may add to the disturbance of postural stability caused by neck pain $(11,13)$. This effect can be seen in Pool et al and Field et al studies (65-82 years compared to 27-30 years), although their methods are similar, different results have been reported $(10,11)$. In some other studies, postural control which assessed in quiet standing, could not reveal postural stability deficiencies in patients with neck pain, possibly because it is a simple well-learned task $(9,10)$. This is more conceivable that dynamic conditions which are more challenging postural tasks might be more sensitive tests to discriminate postural deficit in chronic non-specific neck pain. Nevertheless, to the best of our knowledge, there are no investigations that have examined dynamic postural stability in patients with chronic neck pain.

The aim of this study was to compare the dynamic postural stability between patients with chronic non-specific neck pain and healthy individuals. It was hypothesized that patients with chronic neck pain show decreased dynamic postural stability and this difference are more pronounced in more challenging postural tasks.

\section{Methods}

This was a case-control study conducted in Musculoskeletal Rehabilitation Research Center, Ahvaz Jundishapur University of Medical Sciences, Ahvaz, Iran. Twenty-two patients with a history of nonspecific chronic neck pain were included in this study between April and September 2017. In this study, non-specific neck pain was defined as pain in the cervical region either of mechanical or myofascial origin, provoked by sustained neck postures or specific neck movements without any identifiable diseases or structure anomalies (14). Any specific diagnosis such as malignancy, infection, inflammatory disorder, or fracture in the cervical region was excluded by the physician.

The inclusion criteria were age between 20 to 50 years and history of pain more than 3 months in the cervical region (14). Exclusion criteria for patients were a history of cervical spine surgery, whiplash injury, pregnancy, signs of cervical radiculopathy and pain report in the hip, knee, and ankle except for neck in three months ago. Subjects excluded if they had diabetes, rheumatoid arthritis, vestibular or neurological disorder.(9). In the control group, 22 healthy individuals were matched with people with neck pain based on age, weight, height, and body mass index. The same exclusion criteria were used for participants in the control group. Nineteen females $(86 \%)$ and three males $(13.66 \%)$ participated in each group. The sample size was determined, based on the pilot study information for total stability index variable. The following formula was applied to calculate the sample size $\mathrm{n}=\frac{\left(s_{1}^{2}+s_{2}^{2}\right)^{2}\left(z_{1-\frac{\alpha}{2}}+z_{1-\beta}\right)^{2}}{(\overline{x 1}-\overline{x 2})^{2}}$, where: [neck pain group: $\mathrm{x} 1=9.45, \mathrm{~S} 1=15.21$ and control group: $\mathrm{x} 1=5.30, \mathrm{~S} 2=15.21$ ], a power of $90 \%$, alpha level $=0.05$ which obtained a sample size of 22 subjects with $10 \%$ drop in each group were considered. The study was approved by the ethics committee of Jundishapur University of Medical Sciences, Ahvaz, Iran (Approval number: IR.Ajums.REC.1394.520). All participants signed an informed consent form before participating in the study. They completed a demographic questionnaire and pain intensity and disability information (for the neck pain group) prior to participation. We used visual analog scale for measurement of pain intensity and also, Neck Disability Index for assessment of disability.

\section{Procedure}

Techno-body Prokin tilting platform (Prokin PK254, Technobody Inc, Italy) was used for measurement of dynamic postural stability indexes. Techno-body is a monoaxial platform which consists of 3 strain gauges that were placed under a circular surface of $55 \mathrm{~cm}$ in diameter and has a $20 \mathrm{~Hz}$ sampling frequency (15). Subjects were tested with two levels of postural difficulty including double-leg standing with opened and closed eyes. Subjects were asked to stand barefoot with arms at side and feet $10 \mathrm{~cm}$ apart. Participants were instructed to stay still, look at a screen in front of them and not to talk during the test (16). The system had 1 to 10 instability level (1 was the most instable and 10 was the least instable condition of the platform). Dynamic balance tests were performed at level 10 of instability. The instability level of 10 was determined in the pilot test. Balance on a more unstable platform condition was too difficult for the patients to maintain. Simultaneously, an adjustable belt that included a trunk sensor was placed on the sternum of all subjects to record the trunk angular deviation. The position of the trunk sensor was determined according to the software manual of Prokin system. Every time before beginning the tests, the device was recalibrated. The platform was set up at level 10 and the $10 \mathrm{~cm}$ space between feet was determined on the tilt platform. Each test was repeated 3 times with 3 minutes rest between. The orders of the test conditions were randomized. The parameters for assessment of dynamic balance were in terms of AnteriorPosterior stability index (APSI), Medio-Lateral stability index (MLSI) and total stability index (TSI). The stability index is a dispersion index around the expected value (the vertical or horizontal reference axis) (17). APSI was stability index calculated around the vertical reference axis. MLSI was stability index calculated around the horizontal 
reference axis and TSI was the vector sum between "AP axis stability index" and "ML axis stability index". A higher stability index represented more variability and decreased postural stability.

Stability index $=\sqrt{\frac{\sum_{i=1}^{n}(x i-r) 2}{n}}$

Where: $\mathrm{Xi}$ is the obtained value (in degree), $\mathrm{r}$ is the expected value (in degree) and $n$ is the number of obtained samples. Trunk deviation is a screening index that quantifies dynamic balance. Trunk deviation represented total oscillation of upper trunk in AP and ML directions. A good reliability of this variable has been established in physically active persons (ICCs range: 0.55 to 0.79 ) (18).

\section{Statistical Analysis}

Data were analyzed using SPSS version 24 (SPSS Inc., Chicago, IL). We determined the normal distribution of data by the Shapiro-Wilk test ( $p>0.05)$. Independent sample t-test was employed to compare demographic measures between 2 groups. The average measure of the three trials was obtained for each postural variables. Separate $2 \times 2$ [group (neck pain and control) by postural difficulty (eye open and closed)] mixed-design ANOVAs were used to determine main effects and interactions of these factors for each postural stability variable. Level of statistical significance was set at $\mathrm{p}<0.05$.

\section{Results}

The demographic and clinical features of the two groups are presented in Table 1 . There was no statistically significant difference between two groups in terms of age, height, weight, and body mass index. Table 2 shows the mean and standard deviation of postural parameters. A summary of
ANOVA results is presented in Table 3. Interaction of group by postural difficulty was not significant for any postural parameters. The main effects of group and postural difficulty were statistically significant for APSI, MLSI, and TSI (Table 3). There was a statistically significant difference between two groups: APSI $(\mathrm{F}=48.96, \mathrm{p}<0.001)$, MLSI $(\mathrm{F}=54.31, \mathrm{p}<0.001)$, TSI $(\mathrm{F}=74.76, \mathrm{p}<0.001)$. APSI, MLSI, and TSI measurements were higher in the individuals with chronic non-specific neck pain compared to those in the control group in both eyes open and closed conditions (Table 2). Interaction of group by postural difficulty was significant for trunk sway $(\mathrm{F}=4.37, \mathrm{p}=0.04)$. Further analysis by independent t-test showed that trunk sway of neck pain patients was greater than healthy participants in both eyes open and closed conditions. In the closed eye condition postural parameters increased compared to eyes open in both groups.

\section{Discussion}

The purpose of this study was to assess the dynamic postural stability in non-specific chronic neck pain subjects compared to healthy controls. The results of this study indicated that people with non-specific chronic neck pain had poorer postural stability (higher AP, ML, and total stability indexes) compared with matched healthy controls in all conditions (with eyes opened and closed). This balance deficit may be attributed to altered proprioceptive input from the cervical spine (19). Evaluation Joint position sense shows impaired proprioception acuity in chronic neck pain people (20). Pain, altered muscle activation patterns, fatigue and psychological stresses are factors that may

Table 1. Demographic and clinical characteristics of chronic non-specific neck pain and control groups

\begin{tabular}{|c|c|c|c|}
\hline Variables & $\begin{array}{c}\text { Neck pain group } \\
(\mathrm{n}=22)\end{array}$ & $\begin{array}{c}\text { Control group } \\
(n=22)\end{array}$ & $\mathrm{p}$ \\
\hline & Mean (SD) & Mean (SD) & \\
\hline Age (yr) & $31.73(7.64)$ & $32.09(7.22)$ & 0.87 \\
\hline Height (cm) & $164.95(7.05)$ & $163.31(8.08)$ & 0.47 \\
\hline Weight (kg) & $67.81(15.61)$ & $65.22(11.33)$ & 0.53 \\
\hline Body mass index $\left(\mathrm{kg} / \mathrm{m}^{2}\right)$ & $24.87(5.00)$ & $24.42(3.17)$ & 0.72 \\
\hline Time since disease (Mon) & $21.45(17.47)$ & $N / A^{c}$ & N/A \\
\hline Pain $\left(\right.$ VAS) ${ }^{\mathrm{a}}$ & $7.04(1.58)$ & $\mathrm{N} / \mathrm{A}$ & N/A \\
\hline Disability (NDI) ${ }^{b}$ & $44.14(11.31)$ & N/A & N/A \\
\hline
\end{tabular}

aVAS: visual analog scale, bNDI: Neck Disability Scale: Range of scores is from 0 to 100,cN/A: not applicable

Table 2. Mean and standard deviation of dynamic postural stability variables

\begin{tabular}{|c|c|c|c|c|}
\hline \multirow[t]{2}{*}{ Variables } & \multicolumn{2}{|c|}{$\begin{array}{l}\text { Chronic non-specific } \\
\text { neck pain }\end{array}$} & \multicolumn{2}{|c|}{ Control } \\
\hline & Eye open & Eye close & Eye open & Eye close \\
\hline APSI & $5.65(2.37)$ & $9.82(1.56)$ & $3.18(0.75)$ & $6.79(1.64)$ \\
\hline MLSI & $8.38(2.76)$ & $11.07(1.88)$ & $4.32(1.56)$ & $7.50(1.38)$ \\
\hline TSI & $10.38(3.13)$ & $14.79(2.10)$ & $5.49(1.60)$ & $10.14(1.63)$ \\
\hline Trunk deviation & $33.59(11.25)$ & $33.52(11.15)$ & $25.61(10.40)$ & $22.56(11.45)$ \\
\hline
\end{tabular}

APSI: Anterior-Posterior Stability Index; MLSI: Medial-Lateral Stability Index, TSI: Total stability index.

Table 3. Summary of analysis of variance measures of postural performance: $\mathrm{F}$ Ratios and $\mathrm{p}$ values

\begin{tabular}{lcccccccc}
\hline Independent variable & \multicolumn{2}{c}{ APSI } & \multicolumn{2}{c}{ MLSI } & \multicolumn{2}{c}{ TSI } \\
\cline { 2 - 7 } & F-Ratio & P-Value & F-Ratio & P-Value & F-Ratio & P-Value & F-Ratio & P-Value \\
Main effect & & & & & & \\
Group & 48.96 & $<\mathbf{0 . 0 0 1}$ & 54.31 & $<\mathbf{0 . 0 0 1}$ & 74.76 & $<\mathbf{0 . 0 0 1}$ & 7.76 \\
Postural Difficulty & 143.57 & $<\mathbf{0 . 0 0 1}$ & 113.38 & $<\mathbf{0 . 0 0 1}$ & 163.373 & $<\mathbf{0 . 0 0 1}$ & 5.27 \\
Interaction & & & & & $\mathbf{0 . 0 2}$ & \\
Group $\times$ postural difficulty & 0.50 & 0.48 & 1.49 & 0.22 & 0.43 & 0.51 & 4.37 \\
\hline Significant p-values are presented in bold & & & & &
\end{tabular}


change muscle spindles sensitivity and cervical afferent input and lead to impaired balance in chronic neck pain disorder (7). A mismatch between cervical proprioceptive information and visual and vestibular signals in the central nervous system may be an extended hypothesis for postural control disturbances in this population (8). The pain increased pre-synaptic inhibition of muscle afferents, correspondingly dominancy of nociception over proprioception information during experimental pain can influence on the central modulation of cervical somatosensory input to the postural control system (21). A linear relationship between pain intensity and increased postural sway has been described in non-specific neck pain patients (22). These findings are in line with Pool et al and Uthaikhup et al who found decreased postural stability in chronic neck pain subjects compared to control group. Nevertheless, in these studies, people with neck pain were 65 years or older (11, 13). In normal standing, Michaelson et al (9) and Field et al (10) found no difference in postural sway between whiplash-associated disorders and chronic neck pain. Palmgren et al found no impaired balance in standing Romberg test on the force platform in patients with nontraumatic chronic neck pain (23). It can be related to the ease of testing procedure in these studies (24). Therefore, the results of this study showed that the dynamic tests were able to discriminate balance deficits in patients with non-specific chronic neck pain which may be masked under static conditions.

In the current study, trunk sway was significantly greater in neck pain group compared to healthy controls. This could be attributed to a reduced range of motion of the cervical spine which might lead to extra trunk deviation (25). Increase trunk sway has been reported also in vestibular dysfunction and chronic whiplash injury patients $(25,26)$. In these studies, trunk sway was assessed in the lower back (at L2-3 vertebral body level) but in the present study, upper trunk sway (at T3 vertebral body level) was examined.

This study has several limitations. First, the effect of pain intensity was not controlled as a confounding factor. Chronic non-specific neck patients with higher self-reported pain score displayed increased postural sway (22). Therefore, pain could have an impact on the results. This suggests that in a future study, postural control for these patients should be assessed in a relatively pain-free period for finding long-term motor control changes. Also, we suggested comparing the balance of patients with greater pain intensity to those with less pain. Trunk deviation was not assessed during a functional task and walking with different velocities. It is valuable to measure trunk sway in both stance and gait tasks for future research.

\section{Conclusion}

Our findings demonstrate impairment of postural control in patients with chronic neck pain in comparison with healthy controls. The results suggest that clinicians could benefit from dynamic balance assessment in patients with neck pain and specific intervention programs for improvement postural stability.

\section{Acknowledgements}

This study is part of the Ph.D. thesis of Maryam Saadat. Special thanks to Ahvaz Jundishapur University of Medical
Sciences for the financial support (Grant number: pht9427). We are thankful for subjects participating in this study.

\section{Conflict of Interests}

The authors declare that they have no competing interests.

\section{References}

1. Cohen SP, editor Epidemiology, diagnosis, and treatment of neck pain. Mayo Clinic Proceedings; 2015: Elsevier.p.58-90.

2. Hoy D, Protani M, De R, Buchbinder R. The epidemiology of neck pain. Best Prac Res Clin Rheumatol. 2010; 24(6):783-92.

3. Ylinen J, Salo P, Nykänen M, Kautiainen H, Häkkinen A. Decreased isometric neck strength in women with chronic neck pain and the repeatability of neck strength measurements. Arch Phys Med Rehabil. 2004;85(8): 1303-8

4. Sjölander P, Michaelson P, Jaric S, Djupsjöbacka M. Sensorimotor disturbances in chronic neck pain - range of motion, peak velocity, smoothness of movement, and repositioning acuity. Manual Therap. 2008; 13(2):122-31

5. Bressel E, Yonker JC, Kras J, Heath EM. Comparison of static and dynamic balance in female collegiate soccer, basketball, and gymnastics athletes. J Athletic Train. 2007;42(1):42.

6. Shumway-Cook A, Woollacott MH. Motor control: translating research into clinical practice: Lippincott Williams \& Wilkins; 2007.p.12-89.

7. Kristjansson E, Treleaven J. Sensorimotor function and dizziness in neck pain: implications for assessment and management. J Orthop Sport Phys Therap. 2009;39(5):364-77.

8. Treleaven J. Sensorimotor disturbances in neck disorders affecting postural stability, head and eye movement control. Manual Therap. 2008;13(1):2-11.

9. Michaelson P, Michaelson M, Jaric S, Latash ML, Sjölander P, Djupsjöbacka M. Vertical posture and head stability in patients with chronic neck pain. J RehabilMed. 2003;35(5):229-35.

10. Field S, Treleaven J, Jull G. Standing balance: a comparison between idiopathic and whiplash-induced neck pain. Manual Therap. 2008;13(3):183-91.

11. Poole E, Treleaven J, Jull G. The influence of neck pain on balance and gait parameters in community-dwelling elders. Manual Therap. 2008;13(4):317-24.

12. Ruhe A, Fejer R, Walker B. Altered postural sway in patients suffering from non-specific neck pain and whiplash associated disorder-A systematic review of the literature. Chiropract Manual Therap. 2011;19(1):13.

13. Uthaikhup S, Jull G, Sungkarat S, Treleaven J. The influence of neck pain on sensorimotor function in the elderly. Arch Gerontol Geriatr. 2012;55(3):667-72.

14. Tsakitzidis G, Remmen R, Dankaerts W, Van Royen P. Non-specific neck pain and evidence-based practice. Eur Scient J. 2013;9(3).

15. Cattaneo D, Jonsdottir J. Sensory impairments in quiet standing in subjects with multiple sclerosis. Multipl Scleros J. 2009;15(1):59-67.

16. Karadenizli ZI, Erkut O, Ramazanoglu N, Selda U, Camliguney AF, Bozkurt S, et al. Comparision of dynamic and static balance in adolescents handball and soccer players. Turkish J Sport Exer. 2014;16(1):47-54.

17. Elshazly FA, Elnaggar RK. Integrated intensive proprioceptive and visuomotor rehabilitation program for treatment of spastic diplegic Children. Intl J Med Res Health Sci. 2016;5(8):132-9.

18. Mauch M, Kälin X. Reliability of the ProKin Type B line system (TechnoBody ${ }^{\mathrm{TM}}$ ) balance system. 2011.

19. Jull G, Sterling M, Falla D. Whiplash, headache, and neck pain: research-based directions for physical therapies: Elsevier Health Sciences; 2008.

20. de Vries J, Ischebeck B, Voogt L, van der Geest J, Janssen M, Frens $\mathrm{M}$, et al. Joint position sense error in people with neck pain: a systematic review. Manual Therap. 2015;20(6):736-44.

21. Lederman E. Neuromuscular rehabilitation in manual and physical therapies: principles to practice: Elsevier Health Sciences; 2010.p.1207.

22. Ruhe A, Fejer R, Walker B. On the relationship between pain intensity and postural sway in patients with non-specific neck pain. J Back Musculoskelet Rhabil. 2013;26(4):401-9. 
23. Palmgren PJ, Andreasson D, Eriksson M, Hägglund A. Cervicocephalic kinesthetic sensibility and postural balance in patients with nontraumatic chronic neck pain-a pilot study. Chiroprac Osteopath. 2009;17(1):6.

24. Shiravi Z, Shadmehr A, Moghadam ST, Moghadam BA. Comparison of dynamic postural stability scores between athletes with and without chronic ankle instability during lateral jump landing. Muscl Ligam Tend J. 2017;7(1):119.

25. Sjöström H, Allum JH, Carpenter MG, Adkin AL, Honegger F, Ettlin T. Trunk sway measures of postural stability during clinical balance tests in patients with chronic whiplash injury symptoms. Spine. 2003;28(15):1725-34.

26. Allum JH, Adkin AL, Carpenter MG, Held-Ziolkowska M, Honegger F, Pierchala K. Trunk sway measures of postural stability during clinical balance tests: effects of a unilateral vestibular deficit. Gait Posture. 2001;14(3):227-37. 